\title{
Correction to: Characterization of cyanobacterial allophycocyanins absorbing far-red light
}

\author{
Nathan Soulier ${ }^{1} \cdot$ Tatiana N. Laremore $^{2} \cdot$ Donald A. Bryant $^{1,3}$ (D)
}

Published online: 12 November 2020

๑) Springer Nature B.V. 2020

Correction to: Photosynthesis Research (2020) 145: 189-207
https://doi.org/10.1007/s11120-020-00775-2

In the originally published version of Fig. 4 in Soulier et al., Photosynth. Res. 145:189-207 (2020), the line colors for the absorbance and fluorescence spectra for ApcD2 and ApcB2 were mistakenly reversed. The correct version of Fig. 4 appears below.

Correspondingly, in the Results sub-section "Characterization of heterologously produced APs associated with FaRLiP", paragraph 2, sentence 2, the text should read: "The recombinant ApcD2 and ApcB2 subunits had absorbance maxima at $614 \mathrm{~nm}$, although the former had a substantial shoulder at about $675 \mathrm{~nm}$."

Finally, in the Discussion sub-section "Recombinant FRL-APs", paragraph 1, sentence 3, the text should read: "When only a single gene was expressed and the corresponding protein purified, the doubled or broadened absorbance band(s) of ApcD2, ApcD3, and ApcD5 suggest the formation of homodimers or other oligomers (Fig. 4)."
The original article can be found online at https://doi.org/10.1007/ s11120-020-00775-2.

\section{Donald A. Bryant}

dab14@psu.edu

1 S-002 Frear Laboratory, Department of Biochemistry and Molecular Biology, The Pennsylvania State University, University Park, PA 16802, USA

2 Proteomics and Mass Spectrometry Core Facility, Huck Institute for the Life Sciences, The Pennsylvania State University, University Park, PA 16802, USA

3 Department of Chemistry and Biochemistry, Montana State University, Bozeman, MT 59717, USA 


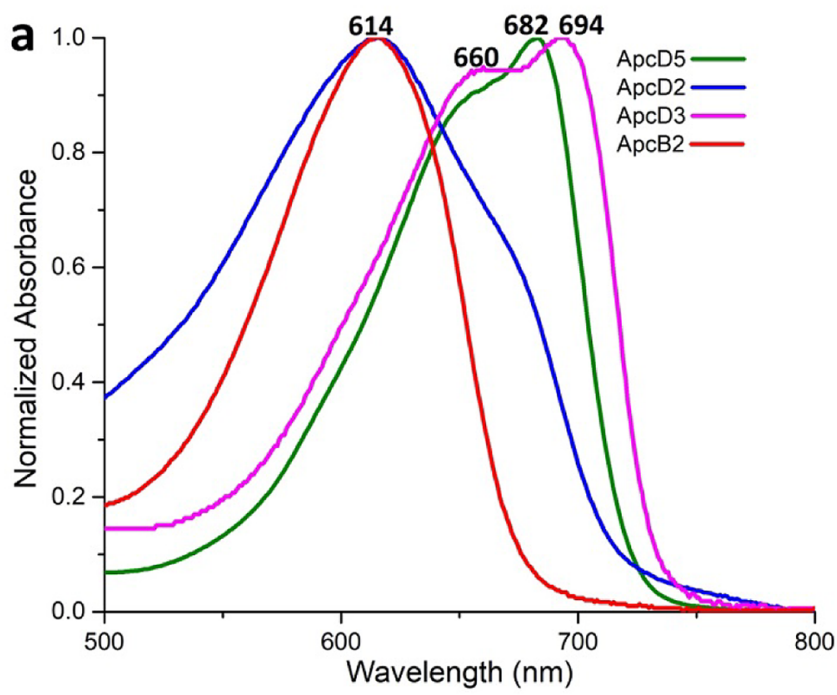

Fig. 4 Spectral properties of FaRLiP AP subunits from Leptolyngbya JSC-1 after heterologous expression in E. coli and purification by IMAC. a The absorbance spectra of individual subunits: ApcD5,

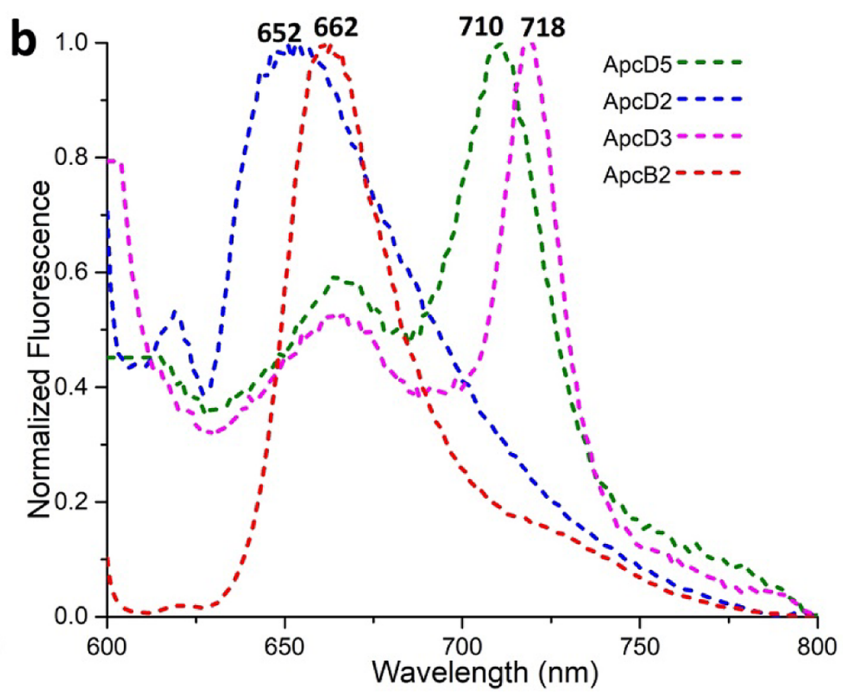

green; ApcD2, blue; ApcD3, magenta; and ApcB2, red. b The 77-K fluorescence emission spectrum of individual subunits $\left(\lambda_{\mathrm{ex}}=590 \mathrm{~nm}\right)$; line colors are the same as in a

Publisher's Note Springer Nature remains neutral with regard to jurisdictional claims in published maps and institutional affiliations. 